\title{
Loss factor for short bunches in azimuthally symmetric tapered structures
}

\author{
A. Blednykh and S. Krinsky \\ Brookhaven National Laboratory, Upton, New York 11973, USA
}

(Received 1 April 2010; published 7 June 2010)

\begin{abstract}
We use the program ECHO to study numerically the loss factor of axisymmetric cavities and collimators with and without tapering. We consider a Gaussian driving bunch of length $\sigma$, and structures with inner radius $b$, outer radius $d$, cavity gap (or collimator inner length) $g$, and taper length $L$. In all cases we consider a short bunch with $\sigma \ll b$. For the untapered structures $(L=0)$, in certain regimes, we describe our numerical results using the known expressions for a step transition and for the diffraction model. In addition, we identify new regimes where $g<\sigma$ and/or $d-b<\sigma$, for which the data must be described by new phenomenological expressions. For a tapered collimator with $d-b>b$, we present a phenomenological formula which accurately describes the dependence of the loss factor on the parameters $\sigma, b, d$, and $L$. This formula also holds for a tapered cavity with $g>d^{2} / \sigma$ and $d-b>b$.
\end{abstract}

DOI: 10.1103/PhysRevSTAB.13.064401

PACS numbers: 41.20.Jb, 41.60. $-\mathrm{m}$

\section{INTRODUCTION}

In the design of the vacuum enclosure for particle accelerators, there is often the need to incorporate a change in cross-sectional geometry. Examples of accelerator components that require changes in cross section are rf cavities to accelerate the beam and collimators to scrape off the beam halo. When a charged particle beam travels through a vacuum chamber with changes in the cross section, electromagnetic fields are generated which can act back on the beam causing it to lose energy and even become unstable. To describe this process, we must calculate the beam impedance [1-3]. In recent years there has been significant progress in both the development of numerical [4-6] and analytical $[7,8]$ methods to calculate the beam impedance.

In this paper, we consider the simplified case of structures with axisymmetric cross section and use the program ECHO $[4,5]$ to calculate the loss factor. We consider a bunch of total charge $Q$ having a Gaussian longitudinal profile with rms length $\sigma$. The loss factor $\kappa_{\text {loss }}$ depends on the bunch length $\sigma$ and is defined to be the energy $\Delta E$ lost by the bunch when it traverses the structure divided by the total bunch charge squared,

$$
\kappa_{\mathrm{loss}}(\sigma) \equiv \Delta E / Q^{2} \text {. }
$$

We shall consider both tapered and untapered structures. An untapered cavity is illustrated in Fig. 1(a) and an untapered collimator in Fig. 1(b). We denote the smaller pipe radius by $b$ and the larger radius by $d$. The gap of the cavity and the length of the inner section of the collimator are denoted by $g$. A tapered cavity is illustrated in Fig. 2(a) and a tapered collimator in Fig. 2(b). The length of the tapered section is denoted by $L$. In all cases that we shall consider, we assume the rms bunch length is short compared to the smallest radius, i.e. $\sigma \ll b$.

Our analysis is based upon studying the dependence of the loss factor on the geometric parameters by carrying out numerical calculations using the ECHO [4,5] code. For the untapered structures, there exist two regimes in which approximate expressions for the loss factor are known. For the untapered collimator [Fig. 1(b)] with $d-b \gg \sigma$ and the cavity [Fig. 1(a)] in the "step regime," specified by $g>d^{2} / \sigma$ and $d-b \gg \sigma$, the loss factor is approximately given by $[2,3,9]$

$$
\kappa_{\mathrm{loss}} \approx \frac{2}{\sqrt{\pi}} \frac{\log (d / b)}{\sigma} .
$$

To get the loss factor in mks units, multiply by $c Z_{0} / 4 \pi$.

For the untapered cavity in the "cavity regime" which we specify by $b<g<b^{2} / \sigma$ and $d-b>\sqrt{g \sigma}$, the diffraction model holds and $[2,3,9,10]$

$$
\kappa_{\mathrm{loss}} \approx \frac{\Gamma(1 / 4)}{\pi \sqrt{\pi}} \frac{1}{b} \sqrt{\frac{g}{\sigma}} .
$$

As discussed in Sec. II, our numerical results are consistent with Eqs. (1.1) and (1.2) under the conditions specified. In addition, for untapered structures with $g$ and/or $d-b$ smaller than $\sigma$, we have identified new regimes for which the data must be described by new phenomenological formulas.

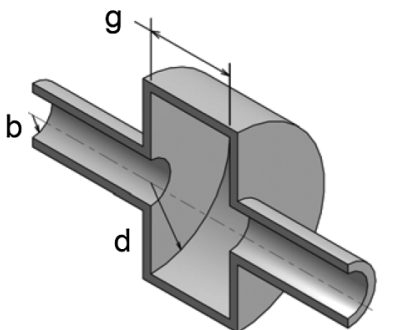

(a)

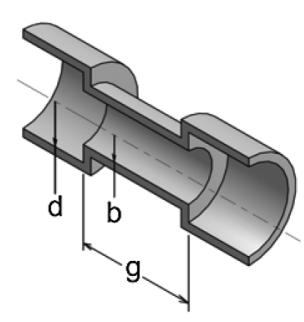

(b)
FIG. 1. (a) Pillbox cavity. (b) Step collimator. 


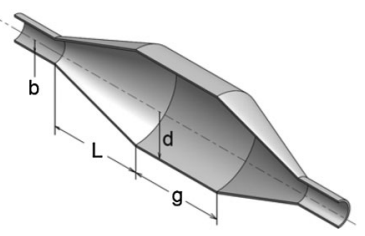

(a)

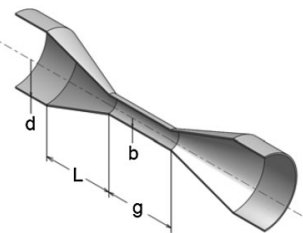

(b)
FIG. 2. (a) Tapered cavity. (b) Tapered collimator.

For tapered structures, we have identified a regime in which a simple approximation provides a good description of the data. For a tapered collimator with $d-b>b$, and for a tapered cavity with $g>d^{2} / \sigma$ and $d-b>b$, the loss factor is well approximated by

$$
\kappa_{\mathrm{loss}} \approx \frac{2}{\sqrt{\pi}} \frac{\log (d / b)}{\sigma}\left[\frac{2}{\pi} \arctan \left(\frac{0.2 d^{2}}{\sigma L}\right)\right]^{2} .
$$

This paper is organized as follows: In Sec. II, we describe the loss factor for untapered cavities and collimators. We note under what conditions the data can be described by the known results for a step transition $[2,3,9]$ and by the diffraction model $[2,3,9,10]$. We also clarify under what conditions new results are required. Phenomenological expressions are presented which describe the data in these new regimes for which $g<\sigma$ and/or $d-b<\sigma$. In Sec. III, we discuss the loss factor for tapered cavities and collimators. For a tapered collimator with $d-b>b$, and for a cavity with $g>d^{2} / \sigma$ and $d-b>b$, the loss factor is shown to be well approximated by the simple expression of Eq. (1.3). In Sec. IV, we present our conclusions.

\section{UNTAPERED STRUCTURES}

\section{A. Pillbox cavity}

We consider a short Gaussian bunch with rms length $\sigma \ll b$. For a pillbox cavity, we specify three regimes: "step," $g>d^{2} / \sigma$; "cavity," $b<g<b^{2} / \sigma$; and "slot," $g<\sigma$. For each of these regimes we present simple formulas which provide good approximations to the results of ECHO calculations. Figures providing comparisons between the formulas and the numerical results are given for each case. The step $[2,3,9]$ and cavity $[2,3,9,10]$ regimes are well known but we are not aware of the slot regime having been identified previously. Moreover, we point out that when $d-b<\sigma$ new asymptotic formulas are required. Equations (2.2), (2.4), (2.5), and (2.6) are new empirical formulas obtained numerically using the ECHO code.

In the step regime, $g>d^{2} / \sigma$,

$$
\kappa_{\mathrm{loss}} \approx \frac{2}{\sqrt{\pi}} \frac{1}{\sigma} \log \left(\frac{d}{b}\right) \quad(d-b \gg \sigma),
$$

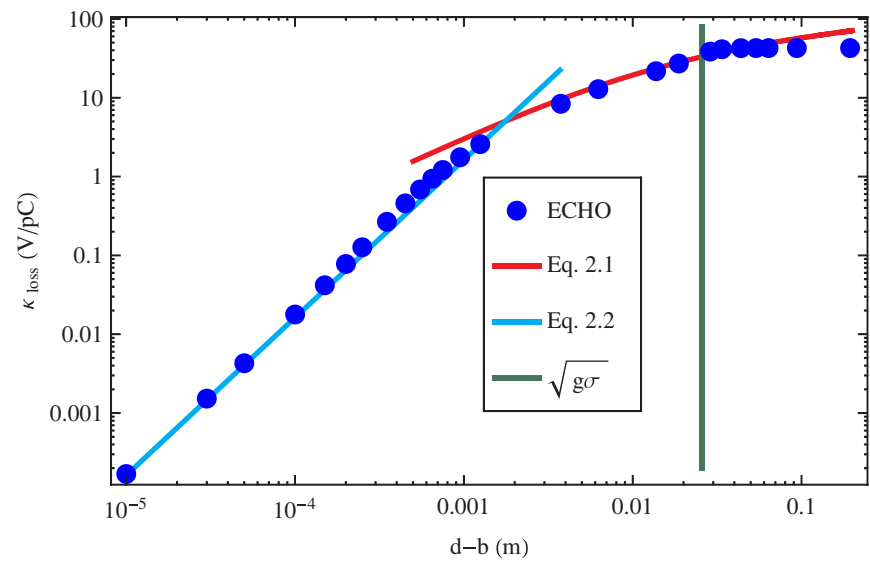

FIG. 3. (Color) For a pillbox cavity in the step regime, the dependence of the loss factor on $d-b$ is shown for $\sigma=$ $0.5 \mathrm{~mm}, b=6.25 \mathrm{~mm}$, and $g=2000 \mathrm{~mm}$. The dots represent the results of calculations using the ECHO code. The red curve is given by Eq. (2.1) and the aqua curve by Eq. (2.2). The vertical green line is $d=\sqrt{g \sigma}$. To the left of this line lies the step regime and to its right the cavity regime.

$$
\kappa_{\mathrm{loss}} \approx \frac{1}{2 \sqrt{\pi}} \frac{1}{b}\left(\frac{d-b}{\sigma}\right)^{2} \quad(d-b<\sigma) .
$$

Equation (2.1) is well known [2,3,9] to hold when $g$ is large enough so that the two steps can be considered to be independent of each other. The dependence of the loss factor on $d-b$ is shown in Fig. 3 and compared with Eqs. (2.1) and (2.2). The good agreement is apparent when $d<\sqrt{g \sigma}$. When $d$ is increased beyond $\sqrt{g \sigma}$, one enters the cavity regime described by Eq. (2.3) below and the loss factor becomes independent of $d-b$. The scaling of the loss factor with $b$ and $\sigma$ given in Eqs. (2.1) and (2.2) has been checked numerically. The dependence of the loss factor on $g$ is discussed later and shown in Figs. 6 and 7.

In the cavity regime, $b<g<b^{2} / \sigma$,

$$
\begin{gathered}
\kappa_{\mathrm{loss}} \approx \frac{\Gamma(1 / 4)}{\pi \sqrt{\pi}} \frac{1}{b} \sqrt{\frac{g}{\sigma}} \quad(d-b>\sqrt{g \sigma}) \\
\kappa_{\mathrm{loss}} \approx \frac{1}{2 \sqrt{\pi}} \frac{1}{b}\left(\frac{d-b}{\sigma}\right)^{2} \quad(d-b<\sigma) .
\end{gathered}
$$

Equation (2.3) is well known $[2,3,9,10]$ to follow from the diffraction model. Equation (2.4) is a new one for a condition when $(d-b<\sigma)$. Note that Eq. (2.4) is the same as Eq. (2.2) given previously for the step regime.

The dependence of the loss factor on $d-b$ is shown in Fig. 4 and compared with Eqs. (2.3) and (2.4). Two lines, red and aqua, fit the numerical data well in specified regions. The loss factor dependence on parameters $b$ and $\sigma$ for Eqs. (2.3) and (2.4) has been checked numerically and compared using several sets of values for parameters within the described regime. The dependence on $g$ is shown in Figs. 6 and 7. 


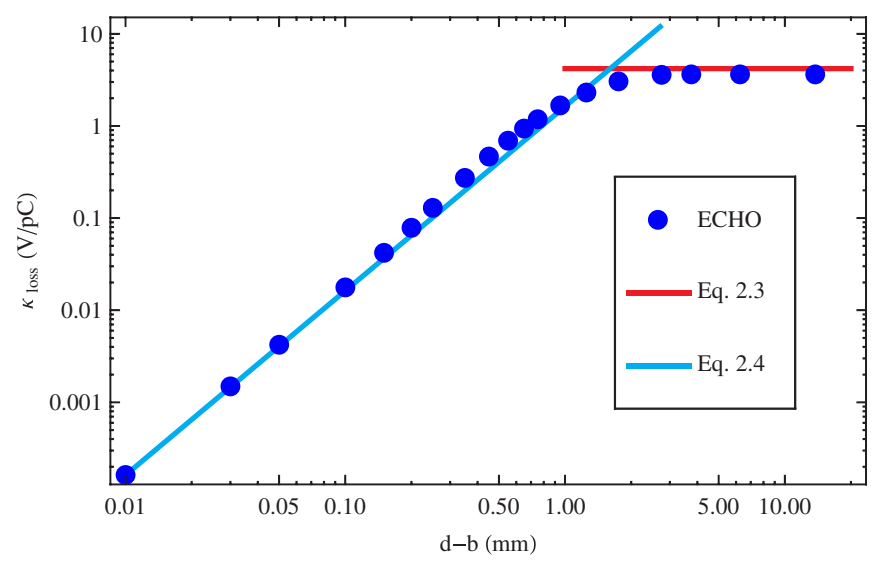

FIG. 4. (Color) For a pillbox cavity in the cavity regime, the dependence of the loss factor on $d-b$ is shown for $\sigma=$ $0.5 \mathrm{~mm}, b=6.25 \mathrm{~mm}$, and $g=10 \mathrm{~mm}$. The dots represent the results of calculations using the ECHO code. The red curve is given by Eq. (2.3) and the aqua curve by Eq. (2.4).

In the slot regime $g<\sigma$,

$$
\begin{gathered}
\kappa_{\mathrm{loss}} \approx \frac{1}{2} \frac{1}{b} \frac{g}{\sigma} \quad(d-b \gg \sigma) \\
\kappa_{\mathrm{loss}} \approx \frac{1}{\sqrt{\pi} \pi} \frac{1}{b}\left(\frac{d-b}{\sigma}\right)^{2}\left(\frac{g}{\sigma}\right)^{2} \quad(d-b<g) .
\end{gathered}
$$

In Fig. 5 we plot the loss factor dependence on $d-b$, simulated numerically (green dots). These data are compared with Eqs. (2.5) and (2.6), describing the loss factor in the slot regime. The scaling of the loss factor on the parameters $g, \sigma, b$, and $d-b$ given by Eqs. (2.5) and (2.6) has been checked numerically.

The upper curve in Fig. 6 illustrates the dependence of the loss factor on $g$ in a case for which $d-b$ is large enough to assure that the three regimes are passed through

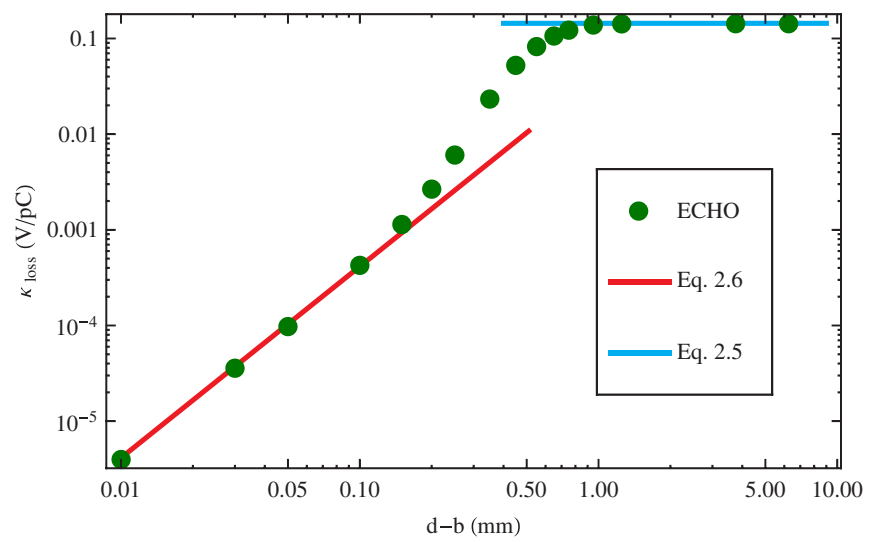

FIG. 5. (Color) For a pillbox cavity in the slot regime, the dependence of the loss factor on $d-b$ is shown for $\sigma=$ $0.5 \mathrm{~mm}, b=6.25 \mathrm{~mm}$, and $g=0.1 \mathrm{~mm}$. The dots represent the results of calculations using the ECHO code. The red curve is given by Eq. (2.6) and the aqua curve by Eq. (2.5).

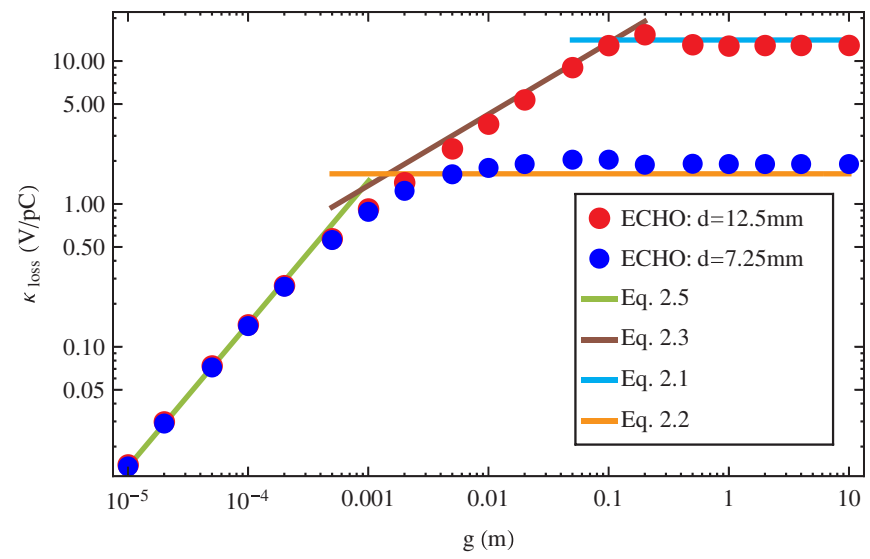

FIG. 6. (Color) For a Gaussian bunch of rms length $\sigma=$ $0.5 \mathrm{~mm}$, we show the results of ECHO calculations of the loss factor as a function of gap $g$ for a pillbox cavity with parameters $d=12.5 \mathrm{~mm}, b=6.25 \mathrm{~mm}$ (red dots) and $d=7.25 \mathrm{~mm}, b=$ $6.25 \mathrm{~mm}$ (blue dots). The green line represents Eq. (2.5) in the slot regime; the brown line represents Eq. (2.3) in the cavity regime; the aqua line represents Eq. (2.1) in the step regime ( $d-$ $b \gg \sigma)$; and the orange line represents Eq. (2.2) in the step regime $(d-b<\sigma)$.

as $g$ is increased: slot $(\sim g)$, cavity $(\sim \sqrt{g})$, and step (independent of $g$ ). In the lower curve, $d-b$ is smaller and there is a transition directly from the slot regime to the step regime as $g$ is increased. Two equations can be applied in the step regime $\left(g>d^{2} / \sigma\right)$. One is the well-known equation (2.1) for $d-b$ large (aqua line in Fig. 6). The other one is Eq. (2.2) for a geometry with $d-b$ small (orange line in Fig. 6). The approximate formulas are seen to provide a good description of the numerical results. In Fig. 7, we illustrate the dependence of the loss factor on $g$

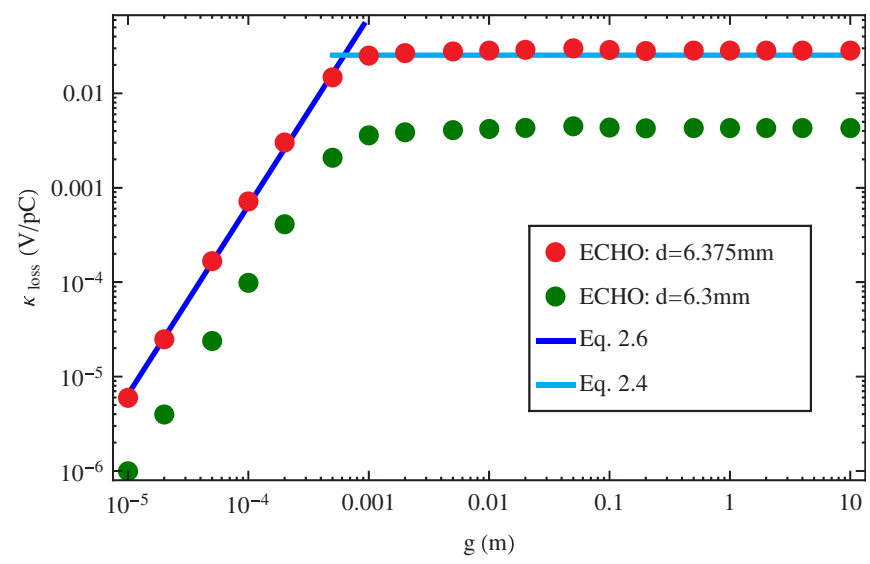

FIG. 7. (Color) For a Gaussian bunch of rms length $\sigma=$ $0.5 \mathrm{~mm}$, we show the results of ECHO calculation of the loss factor as a function of gap $g$ for a pillbox cavity with parameters $d=6.375 \mathrm{~mm}, b=6.25 \mathrm{~mm}$ (red dots) and $d=6.3 \mathrm{~mm}, d=$ $6.25 \mathrm{~mm}$ (green dots). The blue line is given by Eq. (2.6) in the slot regime and the aqua line represents Eq. (2.4) which holds in both the cavity and the step regimes for small $d-b$. 


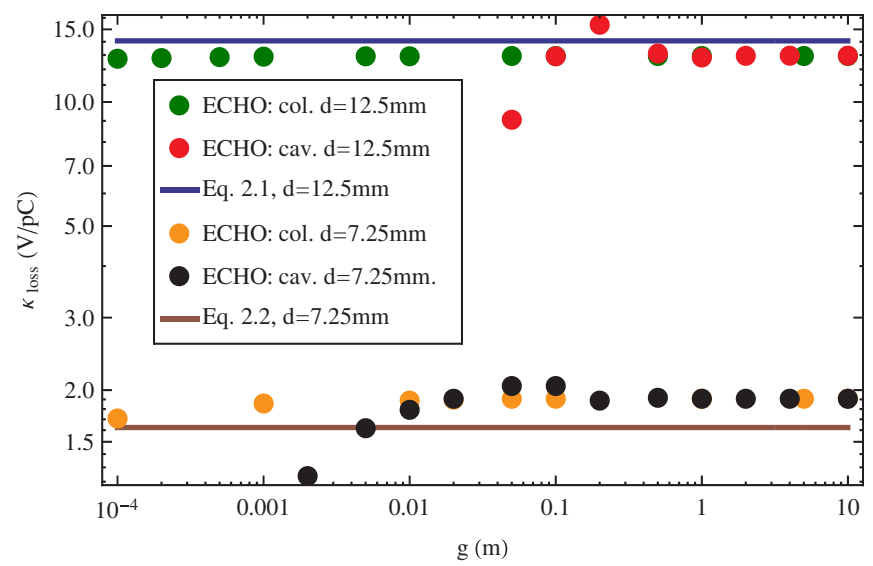

FIG. 8. (Color) For a step collimator and a pillbox cavity, the dependence of loss factor on $g$ for $\sigma=0.5 \mathrm{~mm}, b=6.25 \mathrm{~mm}$ is shown. Red and black dots represent ECHO data already shown in Fig. 6 for a pillbox cavity with $d=12.5 \mathrm{~mm}$ and $d=7.25 \mathrm{~mm}$, respectively. Green and orange dots are the ECHO data corresponding to the collimator structure with parameters $d=$ $12.5 \mathrm{~mm}$ and $d=7.25 \mathrm{~mm}$, respectively. Equation (2.1) (blue line) and Eq. (2.2) (wine line) are also presented.

when $d-b<\sigma$. In this case we pass from the slot regime $\left(\sim g^{2}\right)$ to the step regime (independent of $g$ ).

\section{B. Step collimator}

The dependence on $g$ of the loss factor of a step collimator is shown in Fig. 8, where it is compared with the dependence on $g$ of the loss factor for a pillbox cavity. In Fig. 9, we show the dependence on $d-b$ of the loss factor for a step collimator. The loss factor of the step collimator is seen to be well approximated by Eqs. (2.1) and (2.2), that hold for a pillbox cavity in the step regime (Fig. 3). In the case of a pillbox cavity these equations were valid only for

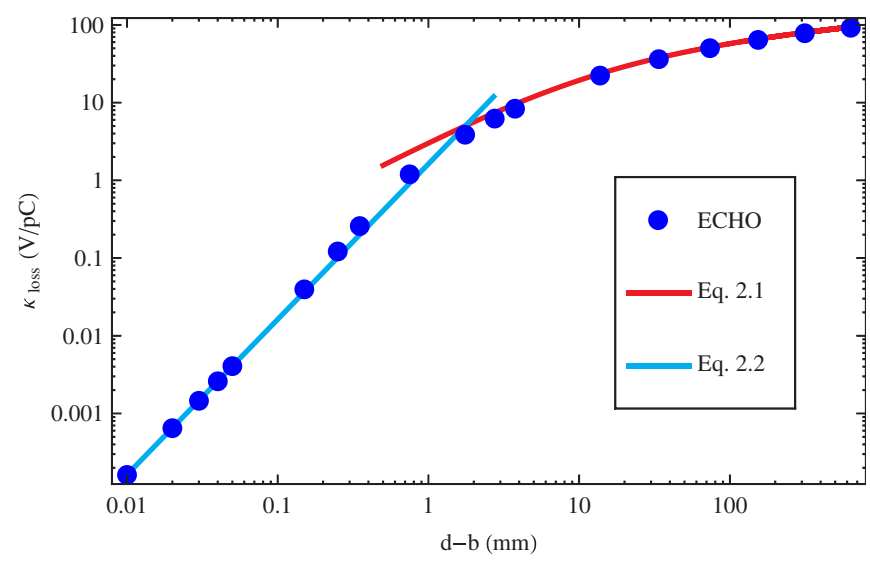

FIG. 9. (Color) For a step collimator, the dependence of the loss factor on $d-b$ is shown for $\sigma=0.5 \mathrm{~mm}, b=6.25 \mathrm{~mm}$, and $g=10 \mathrm{~mm}$. The dots represent the results of calculations using the ECHO code. The red curve is given by Eq. (2.1) and the aqua curve by (2.2). $g>d^{2} / \sigma$; however, for the step collimator they are seen to be good approximations for all $g$.

\section{TAPERED STRUCTURES}

When changes in the vacuum chamber cross section are necessary, it is sometimes possible to reduce the resulting impedance by providing a tapered region between the two different geometries. In the case of axisymmetric collimators with $d-b>b$, and axisymmetric cavities with $g>$ $d^{2} / \sigma$ and $d-b>b$, we have found that the effect of tapering on the loss factor is well described by the simple formula

$\kappa_{\mathrm{loss}} \approx \frac{2}{\sqrt{\pi}} \frac{\log (d / b)}{\sigma}\left[\frac{2}{\pi} \arctan \left(\frac{0.2 d^{2}}{\sigma L}\right)\right]^{2} \quad(d-b>b)$.

The dependence of the loss factor on taper length $L$ is illustrated in Figs. 10 and 11. The good agreement with Eq. (3.1) is apparent. As can be seen from Fig. 10, Eq. (3.1) holds for a geometry with zero taper length as well as for $L \rightarrow \infty$. For $L=0$, Eq. (3.1) becomes equivalent to the well-known equation (1.1). On increasing $L$, the loss factor changes slowly for small $L$, but it drops dramatically $\propto 1 / L^{2}$ for large $L$. This means it is preferable to use long tapers for vacuum components in order to reduce the loss factor significantly when necessary.

In Fig. 11, we plot numerical data for tapered collimators with different parameters $\sigma, d, b$, and fixed $g$, applying a scale factor of $\sigma / \log (d / b)$ to the loss factor and $\sigma / d^{2}$ to the taper length. Analytical data due to Eq. (3.1) are plotted with the same scaling factors (black curve). As one can see, all numerical data lie close to the analytical curve. The agreement is good for all values of $L$.

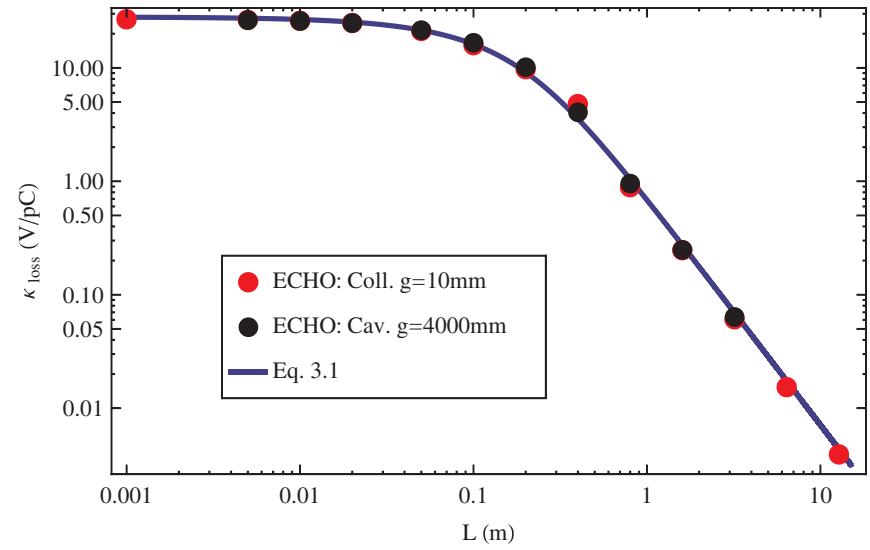

FIG. 10. (Color) We show the results of ECHO calculations of the loss factor as a function of taper length $L$ for a tapered collimator with $g=10 \mathrm{~mm}$ (red dots) and a tapered cavity with $g=4000 \mathrm{~mm}$ (black dots). In both cases $d=25 \mathrm{~mm}, b=$ $6.25 \mathrm{~mm}$, and $\sigma=0.5 \mathrm{~mm}$. The blue curve is given by Eq. (3.1). 


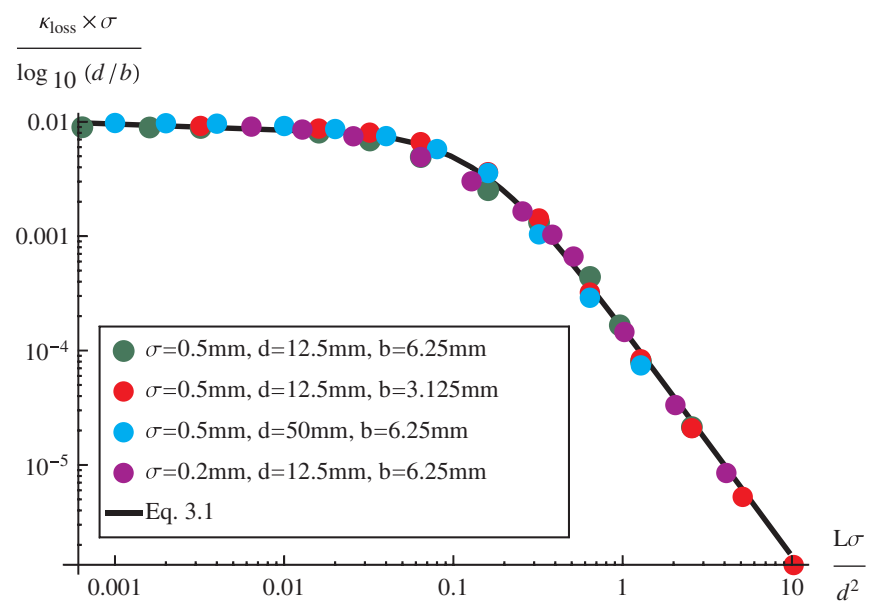

FIG. 11. (Color) For a variety of tapered collimators with $g=$ $500 \mathrm{~mm}$, we plot the scaled loss factor, $\kappa_{\operatorname{loss}} \sigma / \log (d / b)$, $[\mathrm{V}-\mathrm{m} / \mathrm{pC}]$ versus the dimensionless scaled length $L \sigma / d^{2}$. Results of ECHO calculations for the loss factor are shown for parameters: $\sigma=0.5 \mathrm{~mm}, d=12.5 \mathrm{~mm}, b=6.25 \mathrm{~mm}$ (green dots); $\sigma=0.5 \mathrm{~mm}, d=12.5 \mathrm{~mm}, b=3.125 \mathrm{~mm}$ (red dots); $\sigma=0.5 \mathrm{~mm}, d=50 \mathrm{~mm}, b=6.25 \mathrm{~mm}$ (aqua dots); and $\sigma=$ $0.2 \mathrm{~mm}, d=12.5 \mathrm{~mm}, b=6.25 \mathrm{~mm}$ (purple dots). The black curve is given by Eq. (3.1).

Equation (3.1) can be very useful in determination of the loss factors for several types of collimator transitions, which are an important part of the impedance budget for many accelerators.

The loss factor for a tapered collimator $(d-b>b)$ is found to be very insensitive to the length $g$ of the region at the smallest radius. This very weak dependence on $g$ is shown in Fig. 12. Therefore, Eq. (3.1) provides a very good description of the loss factor of a tapered collimator for all $g$.

The dependence of the loss factor for a tapered cavity on the gap length $g$ is not as simple as for the taped collimator.

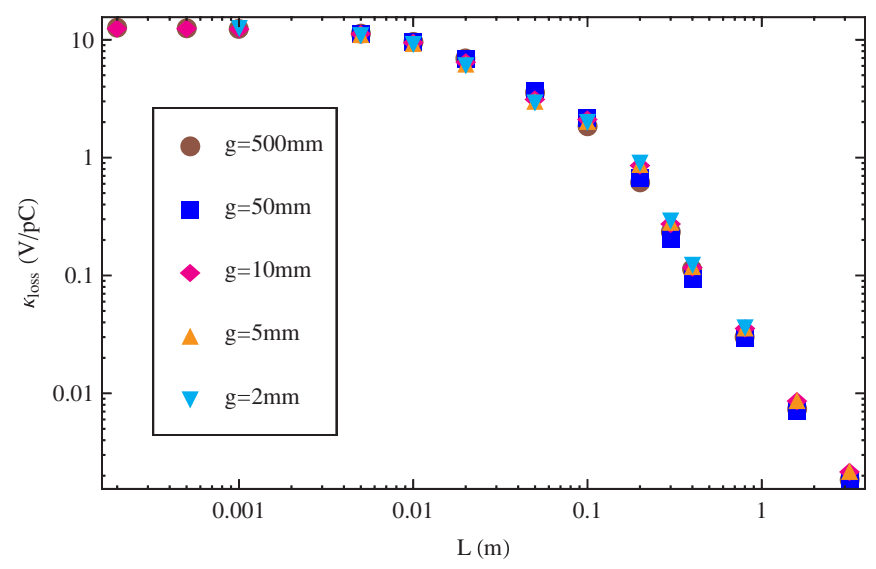

FIG. 12. (Color) For different values of $g$, we show the loss factor as calculated with ECHO as a function of taper length $L$ for the collimator with parameters $d=12.5 \mathrm{~mm}, b=6.25 \mathrm{~mm}$, and $\sigma=0.5 \mathrm{~mm}$.

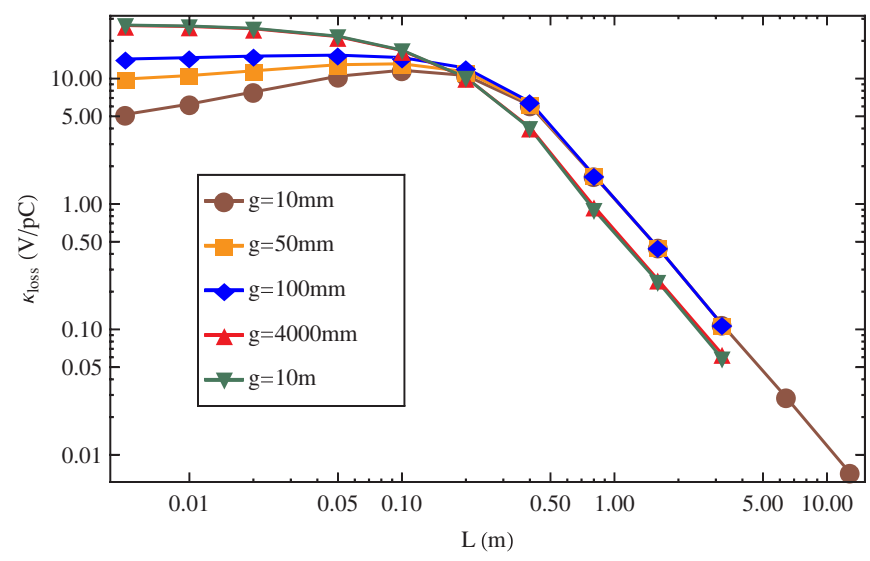

FIG. 13. (Color) For different values of $g$, we show the loss factor as calculated with ECHO as a function of taper length $L$ for the cavity with parameters: $b=6.25 \mathrm{~mm}, d=25 \mathrm{~mm}$ and Gaussian bunch with rms length $\sigma=0.5 \mathrm{~mm}$. The curves are just guides for the eye.

The change in the dependence of the loss factor on taper length $L$ for different $g$ is illustrated in Fig. 13. Therefore, for a tapered cavity, one must be careful to use Eq. (3.1) only for $g>d^{2} / \sigma$, where it applies.

In Fig. 14, we show the dependence of a tapered collimator on $d-b$. For $d-b>b$, it is seen that Eq. (3.1) is in good agreement with numerical calculation. For long tapers $L>d^{2} / \sigma$ and small $d-b<b$, we find that Eq. (3.2) provides a good fit to the data,

$$
\kappa_{\mathrm{loss}} \approx \frac{1}{8 \sqrt{\pi}} \frac{1}{\sigma}\left(\frac{d-b}{\sigma}\right)^{2}\left(\frac{d}{L}\right)^{2} \quad(d-b<b),
$$

as illustrated in Fig. 14. In Fig. 15, the scaled loss factor, $\kappa_{\text {loss }} \times \sigma[\mathrm{V}-\mathrm{m} / \mathrm{pC}]$ is plotted versus the dimensionless scaled length $L \sigma /((d-b) \times d)$ (horizontal axis). When $L>d^{2} / \sigma$, we find that the loss factor is proportional to

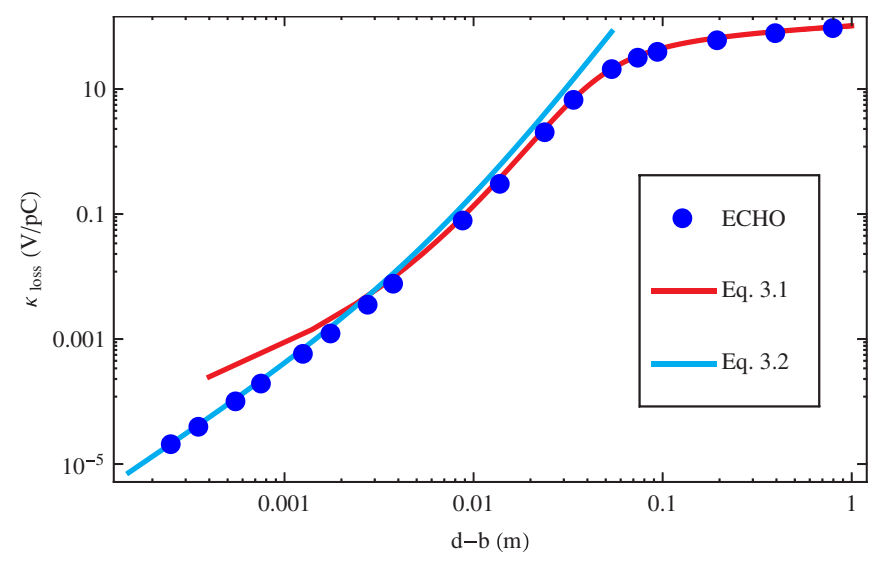

FIG. 14. (Color) ECHO results for the loss factor as a function of $d-b$, for a tapered collimator with $L=800 \mathrm{~mm}, g=500 \mathrm{~mm}$, $b=6.25 \mathrm{~mm}$, and rms bunch length $\sigma=0.5 \mathrm{~mm}$, are shown by blue dots. The red curve is given by Eq. (3.1) and the aqua curve by Eq. (3.2). 


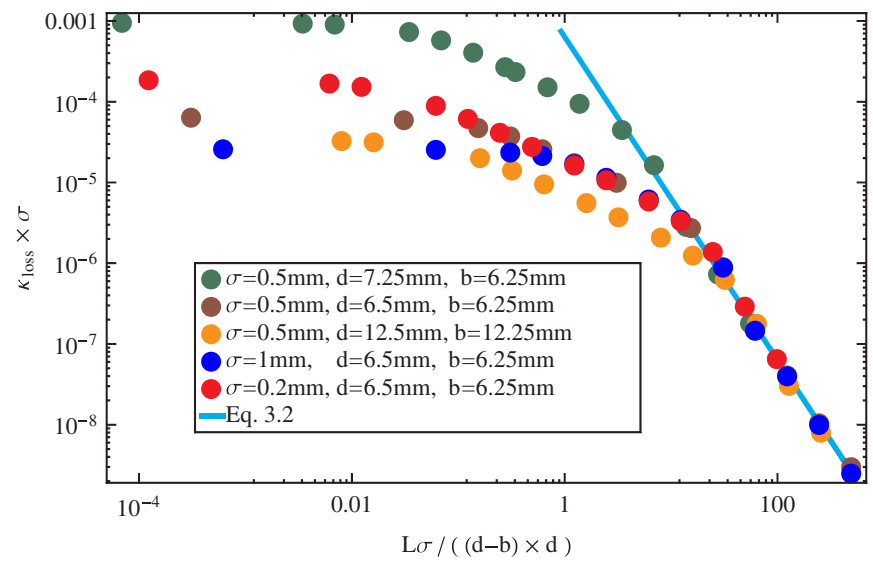

FIG. 15. (Color) The scaled loss factor, $\kappa_{\mathrm{loss}} \times \sigma$, [V-m/pC] versus the dimensionless scaled length $L \sigma /[(d-b) \times b]$ for tapered collimators with $g=500 \mathrm{~mm}$ and various values of $\sigma$, $d$, and $b$. The aqua line is that given by Eq. (3.2).

$1 / L^{2}$ and all numerical data corresponding to geometries with different parameters scale as expected from Eq. (3.2).

\section{CONCLUSIONS}

We have reported the results of numerical calculations using ECHO of the loss factor of axisymmetric cavities and collimators with and without tapering. The spirit of this paper is that of a numerical experimentalist. We try to interpret the numerical results by developing simple formulas describing the data. No analytic work based on Maxwell's equations has been performed to justify the phenomenological relations that we have found. However, one may feel optimistic that, in the future, a theoretical analysis may yield asymptotic relations describing at least some of our numerical results.

We conclude the paper with a summary of our main results. For untapered structures, we have clarified the region of validity of the well-known expressions (1.1) and (1.2) holding in the "step" and "cavity" regimes. We have also presented new phenomenological formulas describing the loss factor for untapered structures having certain dimensions less than the driving rms bunch length. For tapered structures, we have presented a new phenomenological formula (1.3). Within its region of validity, Eq. (1.3) shows excellent agreement with the calculated results from ECHO.

\section{ACKNOWLEDGMENTS}

We thank Dr. I. Zagorodnov for making his ECHO code available for our use and B. Kosciuk for providing us with $3 \mathrm{D}$ rendering geometries. This work was supported by Department of Energy Contract No. DE-AC0298CH10886.

[1] A. W. Chao, Physics of Collective Beam Instabilities in High Energy Accelerators (John Wiley \& Sons, New York, 1993).

[2] B. W. Zotter and S. A. Kheifets, Impedances and Wakes in High-Energy Particle Accelerators (World Scientific, London, 1998).

[3] S. Heifets and S. Kheifets, Rev. Mod. Phys. 63, 631 (1991).

[4] I. Zagorodnov and T. Weiland, Phys. Rev. ST Accel. Beams 8, 042001 (2005).

[5] I. Zagorodnov, Phys. Rev. ST Accel. Beams 9, 102002 (2006).

[6] W. Bruns, GdfidL, http://www.gdfidl.de.

[7] G. V. Stupakov, SLAC Report No. SLAC-PUB-8857, 2001.

[8] G. Stupakov, in Proceedings of the 23rd Particle Accelerator Conference, Vancouver, Canada, 2009 (IEEE, Piscataway, NJ, 2009).

[9] J. J. Bisognano, S.A. Heifets, and B.C. Yunn, in Proceedings of EPAC, Rome, Italy, 1988, Vol. 88, p. 708 [http://accelconf.web.cern.ch/accelconf/].

[10] K. Bane and M. Sands, Part. Accel. 25, 73 (1990). 